\title{
Ferromagnetism and Kondo Insulator Behavior in the Disordered Periodic Anderson Model
}

\author{
Unjong Yu(유운종), ${ }^{1}$ Krzysztof Byczuk, ${ }^{1,2}$ and Dieter Vollhardt ${ }^{1}$ \\ ${ }^{1}$ Theoretical Physics III, Center for Electronic Correlations and Magnetism, Institute of Physics, University of Augsburg, \\ D-86135 Augsburg, Germany \\ ${ }^{2}$ Institute of Theoretical Physics, University of Warsaw, ul. Hoża 69, 00-681 Warszawa, Poland
}

(Received 25 January 2008; published 16 June 2008)

\begin{abstract}
The effect of binary alloy disorder on the ferromagnetic phases of $f$-electron materials is studied within the periodic Anderson model. We find that disorder in the conduction band can drastically enhance the Curie temperature $T_{c}$ due to an increase of the local $f$ moment. The effect may be explained qualitatively and even quantitatively by a simple theoretical ansatz. The emergence of an alloy Kondo insulator at noninteger filling is also pointed out.
\end{abstract}

DOI: 10.1103/PhysRevLett.100.246401

PACS numbers: 71.23. $-\mathrm{k}, 75.20 . \mathrm{Hr}, 75.30 . \mathrm{Mb}$

Materials with $f$-electrons such as the rare earths (e.g., cerium) or actinides (e.g., uranium) exhibit a wealth of highly unusual thermodynamic, magnetic, and transport properties [1]. The minimal microscopic model that can account for this diverse physical behavior is the periodic Anderson model (PAM) which describes a band of noninteracting electrons hybridizing with localized, interacting $f$-electrons [2]. Depending on the position of the $f$-electron energy $\varepsilon^{f}$ relative to the conducting band, and on the strength of the hybridization $V$ and the local Coulomb interaction $U$, the PAM is able to reproduce heavy fermion, intermediate valence, and local moment behavior. At low enough temperatures, the PAM also describes magnetically ordered phases. While antiferromagnetic order is well known to occur close to half-filling [2], ferromagnetic solutions are found far away from halffilling [3]. Indeed, ferromagnetism has been observed in various $f$-compounds [4].

Alloys of $f$-electron materials also display intriguing properties. For example, by changing the stoichiometric composition of $\mathrm{Ce}\left(\mathrm{Pt}_{1-x} \mathrm{Ni}_{x}\right)_{2} \mathrm{Si}_{2}$, the systems can be tuned from the local moment regime at $x=0$ to the intermediate valence regime at $x=1$ [5]. Alloying inevitably introduces disorder into the system. In general, disorder is expected to reduce the tendency towards ferromagnetic longrange order of the $f$-electrons and thus lower the Curie temperature $T_{c}$. On the other hand, in certain cases, disorder is even known to improve the stability of ferromagnetism. For example, disorder in the conduction electron band caused by the substitution of $\mathrm{Rh}$ by $\mathrm{Co}$ in $\mathrm{URh}_{1-x} \mathrm{Co}_{x}$ Ge leads to a maximum in $T_{c}$ at $x \approx 0.6$ [6]. Similarly, a maximum in $T_{c}$ is observed in $\mathrm{CeCu}_{2} \mathrm{Si}_{2-x} \mathrm{Ge}_{x}$ at $x \approx 1.5$ which may be attributed to an enhanced exchange interaction between the $f$-electron moments induced by the diffusive motion of the $\mathrm{Cu}$ electrons [7]. Finally, alloying Ce with $\mathrm{La}$ as in $\left(\mathrm{Ce}_{1-x} \mathrm{La}_{x}\right)_{3} \mathrm{Bi}_{4} \mathrm{Pt}_{3}$ introduces disorder into the $f$-electron system, which can trigger a transition from a Kondo insulator to a dirty metal [8]. Clearly, disorder is an important feature of many $f$-electron alloys and must therefore be included in any comprehensive theoretical study of such compounds.

Previous investigations of the disordered PAM focused, in particular, on the effect of nonmagnetic impurities on the heavy Fermi liquid or the Kondo insulating state [9], and on the disorder-driven non-Fermi liquid behavior in Kondo alloys [10]. Grenzebach et al. [11] recently presented a detailed study of transport properties of the disordered PAM within the dynamical mean-field theory (DMFT) [12] together with a thorough discussion of the development of the field. The effect of disorder in the $f$-electrons on the ferromagnetic phase was investigated by Meyer [13], who found that the Curie temperature is always reduced.

In this Letter, we report results of a detailed study of ferromagnetism in the PAM in the presence of alloy disorder. In particular, we show that $T_{c}$ can be substantially enhanced by disorder in the conduction electrons. We also predict Kondo insulator behavior away from half-filling at particular values of the alloy concentration. Quite generally, the Hamiltonian of the PAM in the presence of local disorder has the form

$$
\begin{aligned}
H= & \sum_{i, j \sigma} t_{i j} c_{i \sigma}^{\dagger} c_{j \sigma}+\sum_{i \sigma}\left(\varepsilon_{i}^{f} f_{i \sigma}^{\dagger} f_{i \sigma}+\varepsilon_{i}^{c} c_{i \sigma}^{\dagger} c_{i \sigma}\right) \\
& +\sum_{i \sigma}\left(V c_{i \sigma}^{\dagger} f_{i \sigma}+V^{*} f_{i \sigma}^{\dagger} c_{i \sigma}\right)+U \sum_{i} n_{i \uparrow}^{f} n_{i \downarrow}^{f},
\end{aligned}
$$

where $c_{i \sigma}^{\dagger}\left(c_{i \sigma}\right)$ and $f_{i \sigma}^{\dagger}\left(f_{i \sigma}\right)$ are creation (annihilation) operators of conduction $(c)$ and localized $(f)$ electrons with spin $\sigma$ at a lattice site $i$. Here the on site energies $\varepsilon_{i}^{f}$ and $\varepsilon_{i}^{c}$ are random variables, and $V$ is the local hybridization between $f$ - and $c$-electrons. The hopping amplitude of the $c$-electrons is given by $t_{i j}$. The Coulomb interaction $U$ acts only between $f$-electrons on the same site.

The alloy will be modeled by a bimodal probability distribution function, $P\left(y_{i}\right)=x \delta\left(y_{i}-y_{0}+\Delta^{y}\right)+(1-$ x) $\delta\left(y_{i}-y_{0}\right)$, where $y_{i}=\varepsilon_{i}^{c}, \varepsilon_{i}^{f}$ are independent, random variables with reference values $y_{0}=\varepsilon_{0}^{c}, \varepsilon_{0}^{f}$. The alloy 
concentration is characterized by the parameter $x$ and the splitting between the atomic levels of the alloy components by the energy $\Delta^{y}=\Delta^{c}, \Delta^{f}$, respectively. While the concentration $x$ and energy splitting $\Delta^{y}$ are, in general, independent parameters, the values $x=0,1$ correspond to a nondisordered system even if $\Delta^{y} \neq 0$. Hence, $\delta^{y}=x(1-$ $x) \Delta^{y}$ is a natural parameter for the disorder strength of alloy disorder.

The PAM with binary alloy disorder is solved within DMFT [12], which becomes exact in the limit of infinite dimensions [14]. In DMFT, the disordered PAM is mapped onto independent impurities; i.e., for each random variable $\left\{y_{i}\right\}$, the action has the form

$$
S^{\mathrm{loc}}\left[f_{\sigma}, c_{\sigma} ;\left\{y_{i}\right\}\right]=\sum_{\sigma n}\left(f_{\sigma n}^{*}, c_{\sigma n}^{*}\right)\left(\begin{array}{cc}
i \omega_{n}+\mu-\varepsilon_{i}^{f} & V^{*} \\
V & i \omega_{n}+\mu-\varepsilon_{i}^{c}-\eta_{\sigma n}
\end{array}\right)\left(\begin{array}{l}
f_{\sigma n} \\
c_{\sigma n}
\end{array}\right)+U \int_{0}^{\beta} d \tau n_{\uparrow}^{f}(\tau) n_{\downarrow}^{f}(\tau) .
$$

The function $\eta_{\sigma n}$ describes an effective dynamical hybridization of the $c$-electrons with the bath. It is the same for all random variables $\left\{y_{i}\right\}$ and is determined by the selfconsistency equations to be discussed below. We start with the local matrix Green function

$$
\mathbf{G}_{\sigma}^{\mathrm{loc}}\left(\tau ;\left\{y_{i}\right\}\right)=-\left(\begin{array}{cc}
\left\langle T_{\tau} f_{\sigma}(\tau) f_{\sigma}^{\dagger}(0)\right\rangle & \left\langle T_{\tau} f_{\sigma}(\tau) c_{\sigma}^{\dagger}(0)\right\rangle \\
\left\langle T_{\tau} c_{\sigma}(\tau) f_{\sigma}^{\dagger}(0)\right\rangle & \left\langle T_{\tau} c_{\sigma}(\tau) c_{\sigma}^{\dagger}(0)\right\rangle
\end{array}\right)
$$

where $T_{\tau}$ is the time-ordering operator. Since the Green function (3) depends on $\left\{y_{i}\right\}$, it is a random function. Here, we perform arithmetic averaging; i.e., the averaged Green function is given by

$$
\mathbf{G}_{\sigma}^{\mathrm{loc}}(\tau)=\int \prod_{\left\{y_{i}\right\}} d y_{i} P\left(y_{i}\right) \mathbf{G}_{\sigma}^{\mathrm{loc}}\left(\tau ;\left\{y_{i}\right\}\right) .
$$

In the absence of interactions, one then obtains the results of the well-known coherent potential approximation (CPA) [15]. Effects of Anderson localization are neglected in this case but can be incorporated by employing the geometric average [16]. The self-consistency requires the averaged local Green function (4) to be the same as the lattice Green function, i.e.,

$$
\mathbf{G}_{\sigma n}=\sum_{\mathbf{k}}\left(\begin{array}{cc}
i \omega_{n}+\mu-\Sigma_{\sigma n}^{f} & V^{*} \\
V & i \omega_{n}+\mu-\epsilon_{\mathbf{k}}-\Sigma_{\sigma n}^{c}
\end{array}\right)^{-1}
$$

The local self-energies appear in the $\mathbf{k}$-integrated Dyson equation $\boldsymbol{\Sigma}_{\sigma n}=G_{\sigma n}^{-1}-\mathbf{G}_{\sigma n}$, where $G_{\sigma n}$ is the local Green function of the noninteracting bath electrons, with

$$
\mathcal{G}_{\sigma n}^{-1}=\left(\begin{array}{cc}
i \omega_{n}+\mu-\varepsilon_{0}^{f} & V^{*} \\
V & i \omega_{n}+\mu-\varepsilon_{0}^{c}-\eta_{\sigma n}
\end{array}\right) .
$$

Equations (2)-(6) form a general, closed set of equations, which determine all local, dynamical correlation functions of the disordered PAM.

To understand the effect of alloy disorder on the physics described by the PAM, it is instructive to investigate the case $U=0$ first since alloy disorder affects a hybridized two-band system in several interesting ways. To this end, we consider the disorder to act only on the $c$-electrons or the $f$-electrons, respectively. In the case of $c$-electron disorder, the diagonal elements of the local Green function (5) are given by

$$
\begin{aligned}
G_{\sigma n}^{c c} & =\frac{x}{\left(G_{\sigma n}^{c c}\right)^{-1}-|V|^{2} G_{\sigma n}^{f f}}+\frac{1-x}{\left(G_{\sigma n}^{c c}\right)^{-1}-|V|^{2} G_{\sigma n}^{f f}-\Delta^{c}} \\
G_{\sigma n}^{f f} & =\frac{x}{\left(G_{\sigma n}^{f f}\right)^{-1}-|V|^{2} G_{\sigma n}^{c c}}+\frac{1-x}{\left(G_{\sigma n}^{f f}\right)^{-1}-\frac{\mid V^{2}}{\left(G_{\sigma n}^{c c}\right)^{-1}-\Delta^{c}}} .
\end{aligned}
$$

The case of $f$-electron disorder is obtained by exchanging $f \leftrightarrow c$ in Eq. (7). Large energy splitting $\Delta^{c}$ leads to a band splitting of the conduction electrons as in the single band model [17]; i.e., each alloy subband contains $2 x N_{L}$ and $2(1-x) N_{L}$ states, respectively, where $N_{L}$ is the number of lattice sites. At the same time, the $f$-level does not split. Altogether, the alloy with hybridized $c$ - and $f$-electrons can therefore be a band insulator even for total densities different from integer values (2 or 4) [17].

We now include the interaction $U$ between the $f$-electrons and investigate its influence on the alloy subbands. The effective two-orbital impurity problem in the presence of disorder, Eqs. (2)-(6), is solved by finite temperature determinant quantum Monte Carlo (QMC) simulations. Ferromagnetic instabilities are detected by the divergence of the static spin susceptibility and by a nonvanishing value of the magnetization [18]. In the numerical examples presented below, the DOS for the noninteracting $c$-electrons has the model form $N_{0}(\varepsilon)=$ $\sqrt{4-\varepsilon^{2}} / 2 \pi$, where the energy unit is $t=1$. In the following, we fix the interaction and the hybridization at $U=1.5$ and $V=0.6$, respectively, and include disorder either in the $f$-electron or $c$-electron system.

As shown in Fig. 1, the computed Curie temperature for the transition to the ferromagnetic state is a nonmonotonic function of the alloy concentration $x$. In particular, the behavior is quite different for disorder acting on the $f$-or the $c$-electrons.

$f$-electron disorder. - In agreement with Meyer [13], the presence of $f$-electron disorder always reduces the Curie temperature relative to its nondisordered values at $x=0$ or 1. For strong enough disorder, $T_{c}$ eventually vanishes, e.g., 


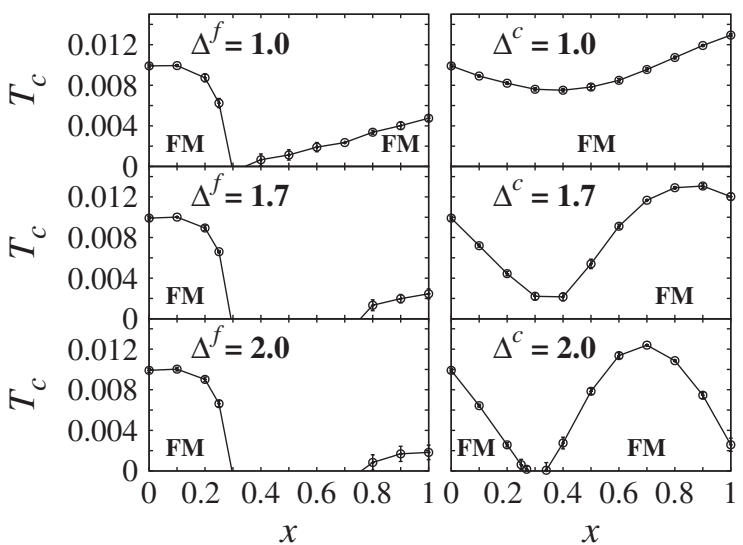

FIG. 1. Curie temperature $T_{c}$ as a function of alloy concentration $x$ and energy splitting $\Delta^{f}$ (left column) and $\Delta^{c}$ (right column) for $n_{\text {tot }}=1.3$ and $\varepsilon_{c}^{0}-\varepsilon_{f}^{0}=3.25$. Strong $c$-electron disorder enhances $T_{c}$ compared to its values at $x=0$ or 1 .

at $x=0.28$ and $x=0.75$, respectively, for $\Delta^{f}=1.7$ (left panel of Fig. 1). This is due to the splitting of the $f$-electron band at large $\Delta^{f}$ which increases the double occupation of the lower alloy subband; this reduces the local moment of the $f$-electrons and thereby $T_{c}$.

c-electron disorder.-By contrast, $c$-electron disorder leads to a much more subtle dependence of $T_{c}$ on concentration $x$. Namely, for increasing energy splitting $\Delta^{c}$, there are, in general, three different features observed, the physical origin of which will be discussed in more detail later: (i) at $x=1$, i.e., in the nondisordered case, $T_{c}$ is reduced; (ii) a minimum develops in $T_{c}$ at $x=n_{\text {tot }}-1>0$; (iii) for sufficiently large $\Delta^{c}$, there exists a range of $x$-values where $T_{c}$ is enhanced over its nondisordered values at $x=0$ or 1 . Altogether, this leads to a global maximum in $T_{c}$ vs $x$. While the decrease of $T_{c}$ at $x=1$ is a simple consequence of the reduction of the energy difference between the $f$-level and the $c$-electron band, $\varepsilon^{c}-\varepsilon^{f}=\varepsilon_{0}^{c}-\varepsilon_{0}^{f}-$ $\Delta^{c}$, for increasing $\Delta^{c}$, the latter effects are more subtle.

To understand the minimum in $T_{c}$ vs $x$, we computed the evolution of the spectral functions $N^{c}(\omega)$ at $x=0.3$ for $\Delta^{c}$ increasing from 0 to 6 (Fig. 2). There is an opening of a gap at the chemical potential signalling a metal-insulator transition in this system. This is caused by the splitting of the $c$-electron band due to binary alloy disorder and the correlations between the $f$-electrons. Namely, for energy splittings $\Delta^{c}$ much larger than the width of the $c$-electron band, the total number of available low-energy states is reduced from $4 N_{L}$ to $[4-2(1-x)] N_{L}=2(1+x) N_{L}$, whereby the filling effectively increases by a factor of $4 /[2(1+$ $x)]$, such that $n_{\text {tot }}^{\text {eff }}=2 n_{\text {tot }} /(1+x)$, if $n_{\text {tot }}<2(1+x)$. For the filling $n_{\text {tot }}=1.3$ studied in the Figs. 1 and 2, the concentration $x=0.3$ is a special case since then $n_{\mathrm{tot}}^{\mathrm{eff}}=$ 2. The system is then effectively at half-filling and behaves as a Kondo insulator at large $U, \Delta^{c}$, and low temperatures. In particular, itinerant ferromagnetism is unfavorable in this case, i.e., $T_{c}=0$ in the vicinity of $x=0.3$ at $\Delta^{c}=2$,

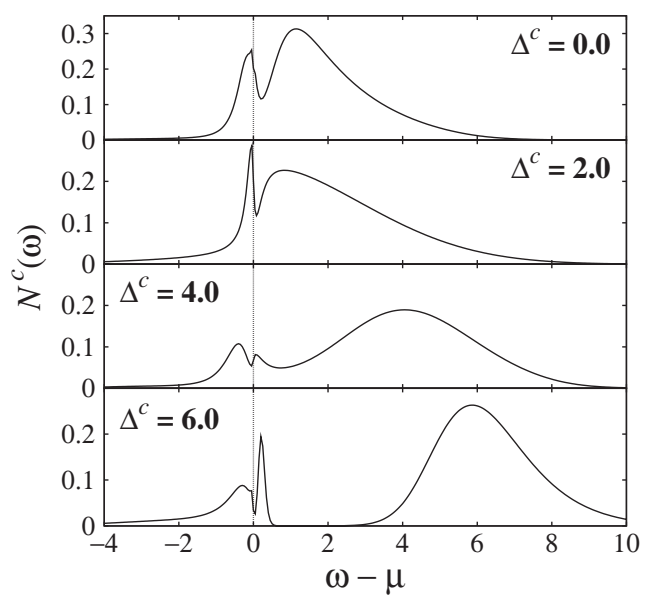

FIG. 2. Spectral function of $c$-electrons for different $\Delta^{c}$ at $x=$ 0.3 (other parameters as in Fig. 1) obtained within QMC calculations and maximal entropy at $T=1 / 60$. By increasing $\Delta^{c}$, a pseudogap opens which becomes a real gap for $T \rightarrow 0$.

cf. Figure 1. The metal to Kondo insulator transition at noninteger filling in the PAM predicted here is a counterpart of the Mott-Hubbard metal to insulator transition at nonintegral fillings in the one-band Hubbard model found in $[17,19]$.

We are now able to understand the maximum in $T_{c}$ vs $x$. It can be explained within the following model based on an ansatz for the Curie temperature, $T_{c}(U, V, \mu)=$ $T_{c}^{0}(U, V, \mu) F^{c}\left(\mu-\varepsilon_{c}^{0}\right) F^{f}\left(\mu-\varepsilon_{f}^{0}\right)$, which implies that the formation of local $f$-electron moments $\left(F^{f}\right)$ is assumed to be independent from the $c$-electron mediated ordering of those moments $\left(F^{c}\right)$. In fact, for the RKKY model, this ansatz can be microscopically justified within a static mean-field theory [20]. The two functions $F^{c}, F^{f}$ are determined by $T_{c}$ calculated within DMFT for the nondisorder case at fixed $\mu-\epsilon_{0}^{c}$ or $\mu-\epsilon_{0}^{f}$, respectively; they are shown in Figs. 3(a) and 3(b) for one set of parameters. The prefactor $T_{c}^{0}$ is determined by the requirement that the dimensionless functions $F^{f}$ and $F^{c}$ be equal to one at their maxima. We note that $F^{f}\left(\mu-\varepsilon_{0}^{f}\right)$ has a maximum when the $f$-level is half-filled $\left(\mu=\varepsilon_{0}^{f}+U / 2\right)$, i.e., when the local moment is maximal.

The Curie temperature in the presence of $c$-electron disorder can now be estimated by averaging over the $c$-electron part, $F^{c}$, giving rise to the disorder-dependent function $\quad \mathcal{F}^{c}\left(x, \mu-\epsilon_{0}^{c}\right)=\left[x F^{c}\left(\mu-\varepsilon_{0}^{c}+\Delta^{c}\right)+(1-\right.$ $\left.x) F^{c}\left(\mu-\varepsilon_{0}^{c}\right)\right]$. The linear dependence on the alloy concentration can again be justified microscopically within a static mean-field theory for the RKKY model, where $T_{c}$ depends linearly on the DOS at the chemical potential [20]. $T_{c}$ is now determined for each concentration $x$. We calculate $\mu$, which is an implicit function of $x$, in the nonhybridized limit $(V=0)$ within a rigid band approximation [21]. The dependence of the resulting functions $\mathcal{F}^{c}(x, \mu-$ $\left.\varepsilon_{0}^{c}\right)$ and $F^{f}\left(\mu-\varepsilon_{0}^{f}\right)$ on $x$ are shown in Fig. 3(c) for 

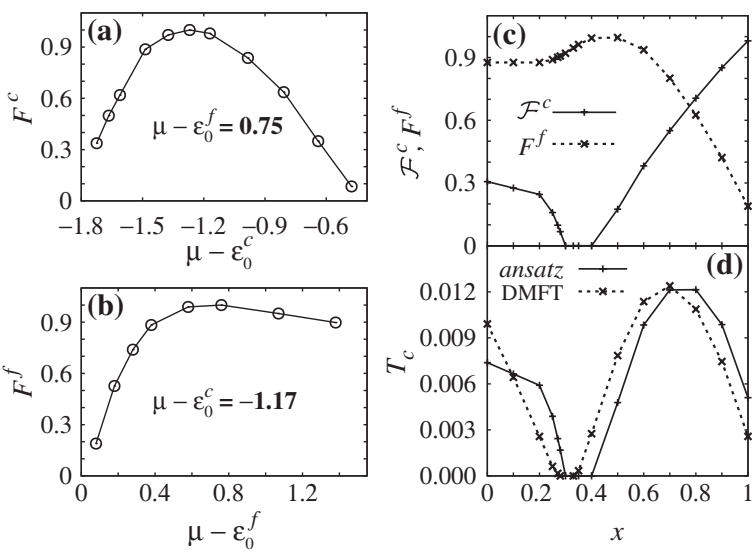

FIG. 3. (a) $F^{c}\left(\mu-\varepsilon_{0}^{c}\right)$, (b) $F^{f}\left(\mu-\varepsilon_{0}^{f}\right)$ appearing in the ansatz for $T_{c}$ (see text) calculated for $\Delta^{c}=0$. (c) $\mathcal{F}^{c}\left(\mu-\varepsilon_{0}^{c}\right)$ and $F^{f}\left(\mu-\varepsilon_{0}^{f}\right)$ for $\Delta^{c}=2.0$; other parameters as in Fig. 1 . (d) Comparison of $T_{c}$ obtained from the ansatz and within DMFT.

$\Delta^{c}=2$.0. In general, $F^{f}\left(\mu-\epsilon_{0}^{f}\right)$ has a maximum at those values of $x$ for which the $f$-level is half-filled [see Fig. 3(c)]. By contrast, $\mathcal{F}^{c}\left(x, \mu-\epsilon_{0}^{c}\right)$ is characterized by a wide minimum, related to the formation of the pseudogap in the interacting DOS seen in Fig. 2. This minimum reaches zero, i.e., $\mathcal{F}^{c}\left(x, \mu-\varepsilon_{0}^{c}\right)=0$, for a finite range of $x$ values as shown in Fig. 3(c). Altogether, the product of $\mathcal{F}^{c}\left(x, \mu-\epsilon_{0}^{c}\right)$ and $F^{f}\left(\mu-\epsilon_{0}^{f}\right)$, which determines the critical temperature, has a global maximum. The resulting $T_{c}(x)$ agrees remarkably well with the numerical result obtained by DMFT as shown in Fig. 3(d).

In conclusion, the interplay between the disorder induced splitting of the conduction band and many-body correlations among the $f$-electrons can lead to a remarkable enhancement of the Curie temperature in the periodic Anderson model. There are two competing effects determining $T_{c}$ as the alloy concentration $x$ is decreased from $x=1$ : (i) a rise due to an increase of the local moment, and (ii) a decrease due to the opening of a gap in the alloy Kondo insulator at noninteger filling. Altogether, this leads to a global maximum in $T_{c}$ vs $x$. Therefore, experimental investigations of $f$-electron materials with alloy disorder in the conducting band are expected to be particularly rewarding.

This work was supported in part by the Sonderforschungsbereich No. 484 of the Deutsche Forschungsgemeinschaft (DFG).

[1] C. M. Varma, Rev. Mod. Phys. 48, 219 (1976); G. R. Stewart, Rev. Mod. Phys. 56, 755 (1984); 73, 797 (2001); L. Degiorgi, Rev. Mod. Phys. 71, 687 (1999).
[2] P. A. Lee, T. M. Rice, J. W. Serene, L. J. Sham, and J. W. Wilkins, Comments Condens. Matter Phys. 12, 99 (1986).

[3] A. N. Tahvildar-Zadeh, M. Jarrell, and J. K. Freericks, Phys. Rev. B 55, R3332 (1997); R. Doradziński and J. Spałek, Phys. Rev. B 56, R14239 (1997); D. Meyer and W. Nolting, Eur. Phys. J. B 18, 385 (2000); C.D. Batista, J. Bonča, and J. E. Gubernatis, Phys. Rev. B 68, 214430 (2003).

[4] See, e.g., T. Durakiewicz, C. D. Batista, J. D. Thompson, C. G. Olson, J. J. Joyce, G. H. Lander, J. E. Gubernatis, E. Guziewicz, M.T. Butterfield, A. J. Arko, J. Bonča, K. Mattenberger, and O. Vogt, Phys. Rev. Lett. 93, 267205 (2004).

[5] F. C. Ragel and P. de V. du Plessis, J. Phys. Condens. Matter 16, 2647 (2004).

[6] S. Sakarya, N. T. Huy, N.H. van Dijk, A. de Visser, M. Wagemaker, A.C. Moleman, T. J. Gortenmulder, J. C.P. Klaasse, M. Uhlarz, and H. v. Löhneysen, arXiv:cond-mat/ 0609557.

[7] M. B. Silva Neto, A. H. Castro Neto, D. Mixson, J. S. Kim, and G. R. Stewart, Phys. Rev. Lett. 91, 257206 (2003).

[8] M. F. Hundley, P.C. Canfield, J.D. Thompson, Z. Fisk, and J. M. Lawrence, Physica B (Amsterdam) 171, 254 (1991).

[9] A. Yoshimori and H. Kasai, Solid State Commun. 58, 259 (1986); Zh.-Zh. Li and Y. Qiu, Phys. Rev. B 43, 12906 (1991); T. Mutou, Phys. Rev. B 64, 245102 (2001); S. Wermbter, K. Sabel, and G. Czycholl, Phys. Rev. B 53, 2528 (1996); P. Schlottmann, Phys. Rev. B 46, 998 (1992).

[10] E. Miranda, V. Dobrosavljević, and G. Kotliar, Phys. Rev. Lett. 78, 290 (1997).

[11] C. Grenzebach, F. B. Anders, G. Czycholl, and Th. Pruschke, Phys. Rev. B 77, 115125 (2008).

[12] A. Georges, G. Kotliar, W. Krauth, and M. J. Rozenberg, Rev. Mod. Phys. 68, 13 (1996); G. Kotliar and D. Vollhardt, Phys. Today 57, No. 3, 53 (2004).

[13] D. Meyer, Solid State Commun. 121, 565 (2002).

[14] W. Metzner and D. Vollhardt, Phys. Rev. Lett. 62, 324 (1989);

[15] R. Vlaming and D. Vollhardt, Phys. Rev. B 45, 4637 (1992); V. Janiš and D. Vollhardt, Phys. Rev. B 46, 15712 (1992).

[16] K. Byczuk, W. Hofstetter, and D. Vollhardt, Phys. Rev. Lett. 94, 056404 (2005).

[17] K. Byczuk, W. Hofstetter, and D. Vollhardt, Phys. Rev. B 69, 045112 (2004).

[18] M. Ulmke, Eur. Phys. J. B 1, 301 (1998); K. Byczuk and D. Vollhardt, Phys. Rev. B 65, 134433 (2002).

[19] K. Byczuk, M. Ulmke, and D. Vollhardt, Phys. Rev. Lett. 90, 196403 (2003).

[20] See, e.g., T. Dietl, J. Cibert, D. Ferrand, and Y. Merle d'Aubigné, Mater. Sci. Eng. B 63, 103 (1999).

[21] Approximating the chemical potential by $\mu=x \mu_{x=1}+$ $(1-x) \mu_{x=0}$ leads to qualitatively similar results as long as $\Delta^{c}$ is smaller than the bandwidth. 